\title{
RADIATION POLLUTION AND ITS IMPACT ON LIVING CREATURES
}

\author{
Revanasiddappa $\mathrm{M}$ \\ Professor, Dept. of Engineering Chemistry \\ PES University Electronic City Campus \\ Bangalore, Karnataka, India
}

\author{
Amarnath B R \\ B.E. Dept. of Electronics \& Communication Engineering \\ PES University, Electronic City Campus \\ Bangalore, Karnataka, India \\ Harish R \\ B.E. Dept. of Computer Science \& Engineering \\ PES University Electronic City Campus \\ Bangalore, Karnataka, India
}

Abstract- Radiation is the phenomena of emission or transmission of energy in the form of waves or in the form of particles. These radiations are emitted through space or through a medium of channel. Ever since the past decade there has been a dramatic increase in the population of wireless transmitters found in the world, with the inevitable result being a dramatic increase in radiofrequency noise pollution. Each electronic device leaks a certain amount of radiation to some level and it is often noticed that the number of cell phones in use these days and their circulation outnumber the number of people. Interference usually results in downgraded performance. For instance, when you are using your electronic gadgets a signal might impact your system or device causing a coverage issue, there might be a reception issue or even an accessing issue. In simple terms interference deals with unwanted energy and it is undesirable when using our electronic gadgets. These days radio systems have spectrum analysis tools built in them. These tools are very helpful in characterizing the signals but they analyse the context of the problem only from a protocol kind of perspective. They provide limited information with regard to the spectrum of a signal channel. In order to understand the problems better in the spectrum, we must use a Real time spectrum analyser (RTSA). This type of analyser is capable of measuring a broader spectrum portion detecting problems within the spectrum as well as in the adjacent ones. In order to study all these phenomena, we have used a new class of batterypowered, USB-based RTSAs such as the Tektronix RSA306 which acts as a powerful tool for interference-

\author{
Prajwal Y \\ B.E. Dept. of Electronics \& Communication Engineering \\ PES University, Electronic City Campus \\ Bangalore, Karnataka, India \\ Sreenivas K \\ B.E. Dept. of Electronics \& Communication Engineering \\ PES University Electronic City Campus \\ Bangalore, Karnataka, India
}

hunting applications. We have collected the data at various places of Bangalore city. This data is useful in order to study the amount of radiation pollution in those places

Keywords- Radiation pollution, Wireless transmitters, radiofrequency noise pollution, Real time spectrum analyzer

\section{INTRODUCTION}

With the advent of technology man has found himself in a dilemma, whether to appreciate the knowledge he possesses or to regret the malign actions that have come from his technological advances. Radiation is one such a dilemma. Man created mobiles and electronic devices that serve his purpose but in doing so has not seen the ill-effects of his creation. Today with the innovative $4 \mathrm{G}$ and $5 \mathrm{G}$ man has failed to realize that nature and humankind are both affected by his innovations. The ability to operate the nearby circuits without any influence is called Electromagnetic compatibility (EMC).This idea is the essential pinnacle of enabling device independence with respect to electromagnetic waves transmission.

Every single electronic circuit however advanced in today's world suffers from the problem of absorbing adjacent electromagnetic radiations. Electromagnetic compatibility (EMC), the technique incorporated in these electronic devices enables these devices to operate without interfering with the EM waves transmitted by other nearby devices. Military usage was the pioneer, the cause when mankind saw the immense yet subtle damage radiation emitted by devices. Nuclear 
weapon was a huge effect of electronic pulse produced by their explosion and high-powered radar systems.

The device and the nature being damaged was the first implication of these weapons. During the 1970s Boolean Logic grew its way into electronics and thus this idea enabled devices to be operated at high clock speeds. However, these advents made this circuitry susceptible to the effects of EMI, and the need for EMC precautions to be integrated into the device grew by folds for the safe and essential working of the devices.

\section{Pollution DuE To EM waVES, EMF AND EMI}

The unnecessary radiation that causes the interference of the working of the other nearby circuitry is called the Electromagnetic interference (EMI). The coupling path measurement and the waveform-switching in the path is used to measure the impact of these radiation on a vulnerable victim.EMI can be superficially classified by the below headers:

a) Continuous interference- An oscillator signal and a radio, two frequently persistent signals are prime examples of the continuous interference. It may be in the form of wideband noise or even from an unscreened oscillator

b) Impulse interference- A phenomenon where the interference is caused due to impulses in non-periodic intervals of time. Activation of a circuit, switching on of a switch and various another daily-life phenomenon falls under this category.

A possible solution is the utilization of the 'spread spectrum technique where the radiation is spread over a wider range of frequency i.e. a higher bandwidth after the modulation of the signal. Spread Spectrum is a careful and controlled modulation of the clock signal in such a way that it does not contribute significantly to jitter. As the bandwidth increases thus the users also benefit with a larger array of signals to resonate thus leading to a loop of sequences.

\section{SignifiCANCE OF RADIATION POLLUTION}

The collection of data for the measurement of the impact to the public due to the harmful radiation is a tedious process. Yet scientists all over the world have highlighted the need to critically review the ill-effects of the radiation phenomenon. Upon the advent of $5 \mathrm{G}$, it is realized that such high bands have a 'subtle yet prominent impact' on mankind.

Many recent scientific publications have shown that EMF affects living organisms at levels well below most international and national guidelines. Some of those effects are increased cancer risk, increase in harmful free radicals, neurological disorders, cellular stress, structural and functional changes of the reproductive system, genetic damages, learning and memory deficits, and negative impacts on general wellbeing in humans. Not only humans, but also animals and plants too are being victim of these harmful radiations.

The importance of this study is shown by The International EMF Scientist and they also propose the need for a stronger

exposure limit restriction. $5 \mathrm{G}$ is going to use spectrum in the existing LTE frequency range, i.e. $600 \mathrm{MHz}$ to $6 \mathrm{GHz}$, and also in millimeter wave bands i.e. $24-86 \mathrm{GHz}$. This extremely high frequency has shown (Manoj et al. 2016; TRAI, 2014) that the EMF pollution is slowly going to damage the ecosystem and cause severe implications to mankind.

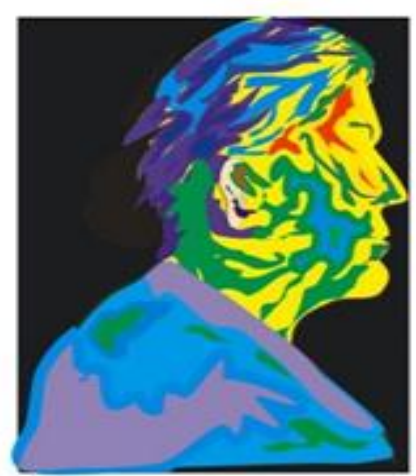

Thermographic image of the Face and Head with no exposure to harmful mobile phone radiation.

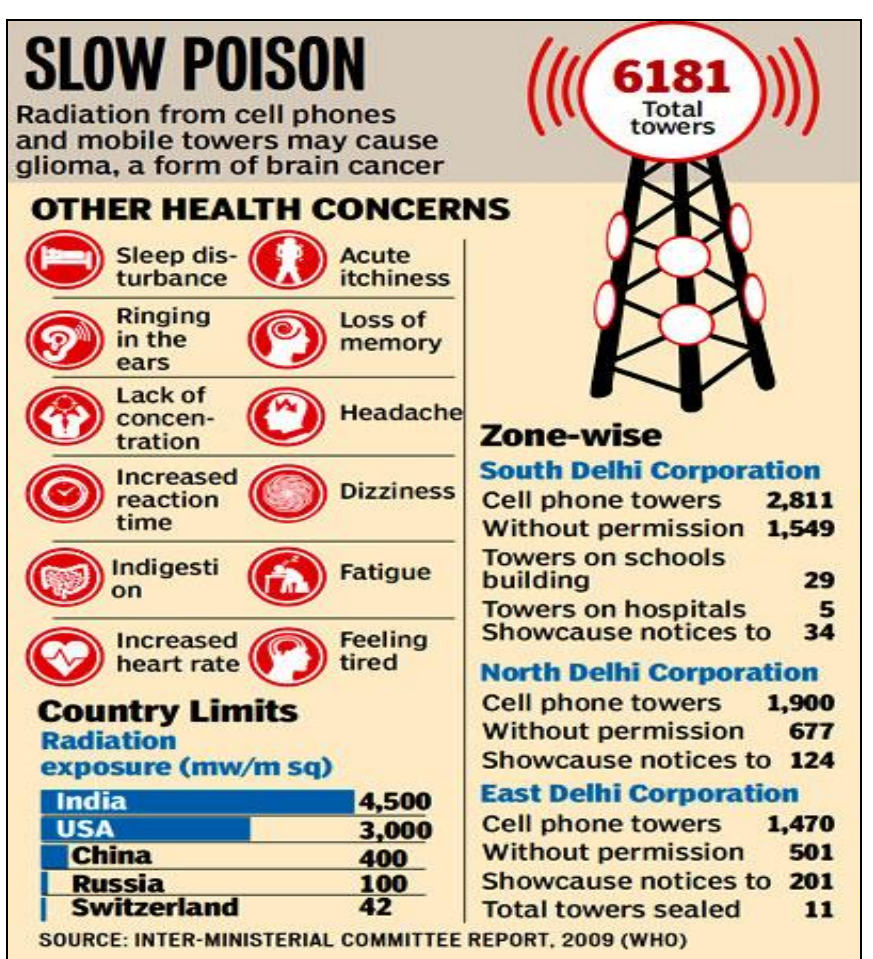

Fig.2: An article on radiation effect

The Real Spectrum Analyzer- The electromagnetic radiation can be of short range as well as longer range. In our case study we wanted to show that there is an EMR being emitted by the electronic devices around us. So, a Real Time Spectrum Analyzer (RSTA) can be used in order to measure 


\section{International Journal of Engineering Applied Sciences and Technology, 2020 \\ Vol. 5, Issue 4, ISSN No. 2455-2143, Pages 437-441 \\ Published Online August 2020 in IJEAST (http://www.ijeast.com)}

this intensity of EMR .Real-time Spectrum Analyzer which is the acronym for RTSA is a recent method which uses overlapping Fast-Fourier transforms (FFTs) and high-speed memory to give a 100 percent probability of intercept even when it is an extremely dense environment. Real-time bandwidth, which is the maximum frequency span offering gap-free overlapping FFT, is an important varying parameter of a Real-time Spectrum Analyzer which can be enabled for more detailed analysis of a spectrum (https://signalhound.com/news/what-you-need-to-know-aboutreal-time-spectrum-analysis). We have used the 'Tektronix RSA306 real-time spectrum analyzer' and 'Signal Vu-PC'M software' which is provided by the Tektronix. A full-featured spectrum analysis and deep signal analysis can be performed by the Tektronix RSA306. The RSA306 is very unique device and it separates signal acquisition from measurement which dramatically lowers even the cost of instrument hardware. Data analysis, storage and replay can be performed on the PC, tablet or laptop.

Managing the personal computer separately from the acquisition of hardware makes processing upgrades easy, and minimizes IT management issues (https://in.tek.com/datasheet/rsa306-usb-real-time-spectrumanalyzer-datasheet-0). Signal Vu-PC is a powerful program that is the basis of Tektronix performance signal analyzers. The RSA306 uses this software since Signal Vu-PC offers a deep analysis capability which was previously unavailable in value-priced solutions (https://in.tek.com/datasheet/rsa306usb-real-time-spectrum-analyzer-datasheet-0). The real-time processing of DPX spectrum is also enabled by this software onto the PC which further reduces the cost of hardware (https://ntp.niehs.nih.gov/).

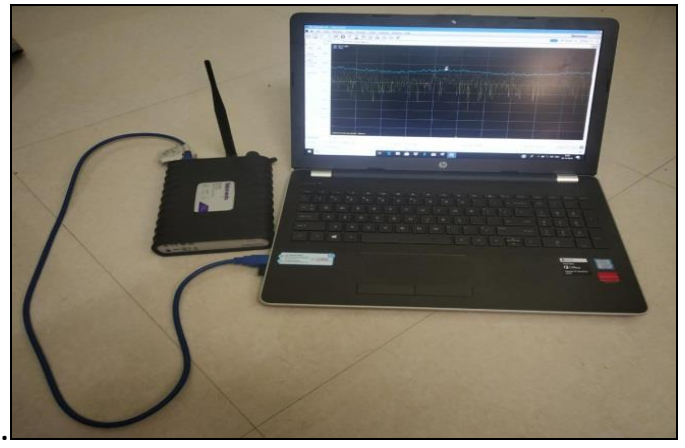

Fig. 3: RSA306 and Signal Vu software

\section{THE CASE OF RADIATION POLLUTION}

A weak radiation source which is close by to us produces a larger electromagnetic field when compared to the strong radiation source which is far from us. Hence, we will experience the strongest EMF which could probably be radiated by the electronic devices in our house like digital phone, computer or any house hold appliance (Indira et al. 1989). In order to measure the intensity of these electromagnetic fields at different places in Bangalore, we used the 'Tektronix RSA306 real-time spectrum analyzer' and 'Signal Vu-PCTM software'. The following are the results:
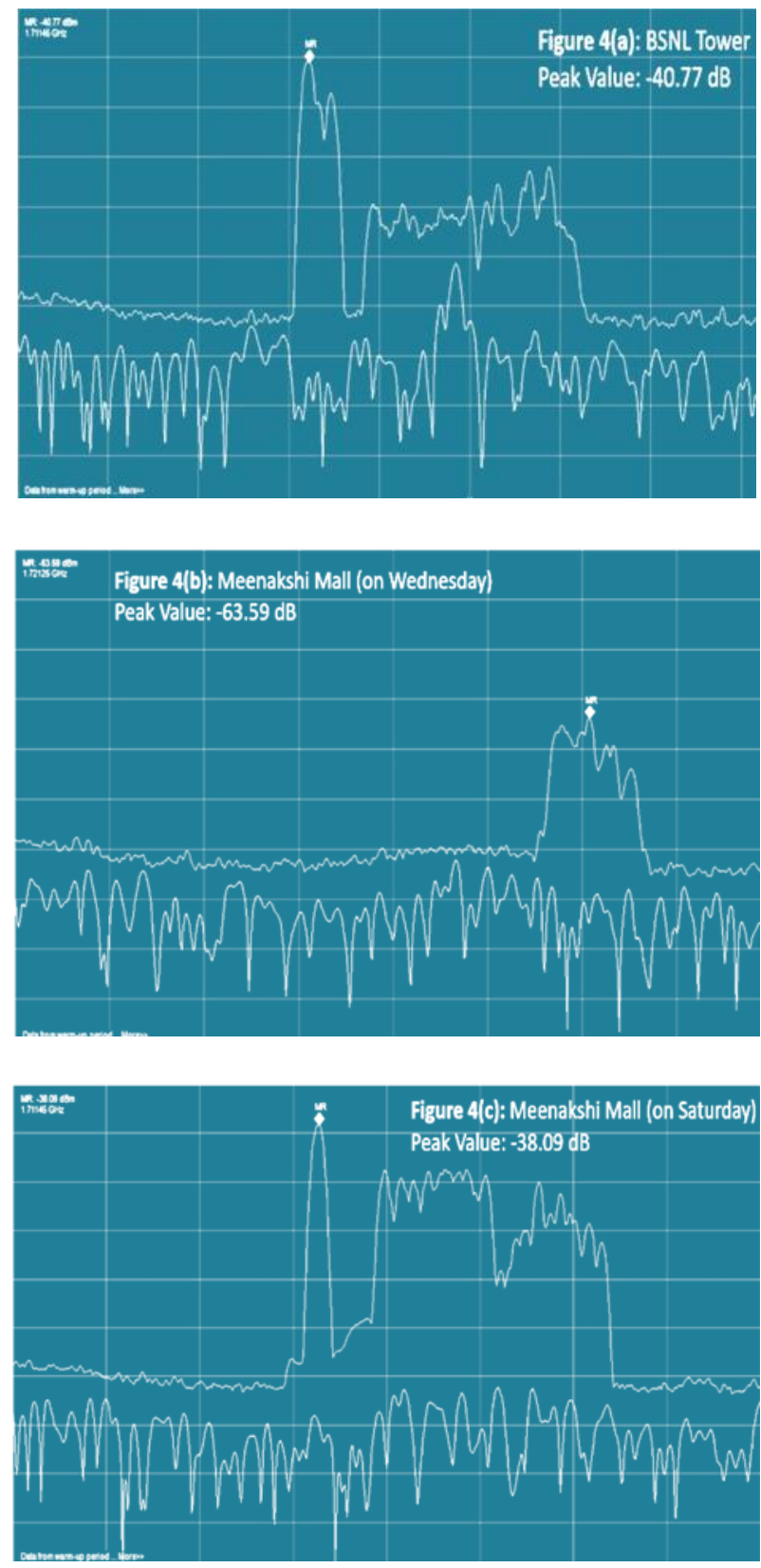
International Journal of Engineering Applied Sciences and Technology, 2020

Vol. 5, Issue 4, ISSN No. 2455-2143, Pages 437-441

Published Online August 2020 in IJEAST (http://www.ijeast.com)
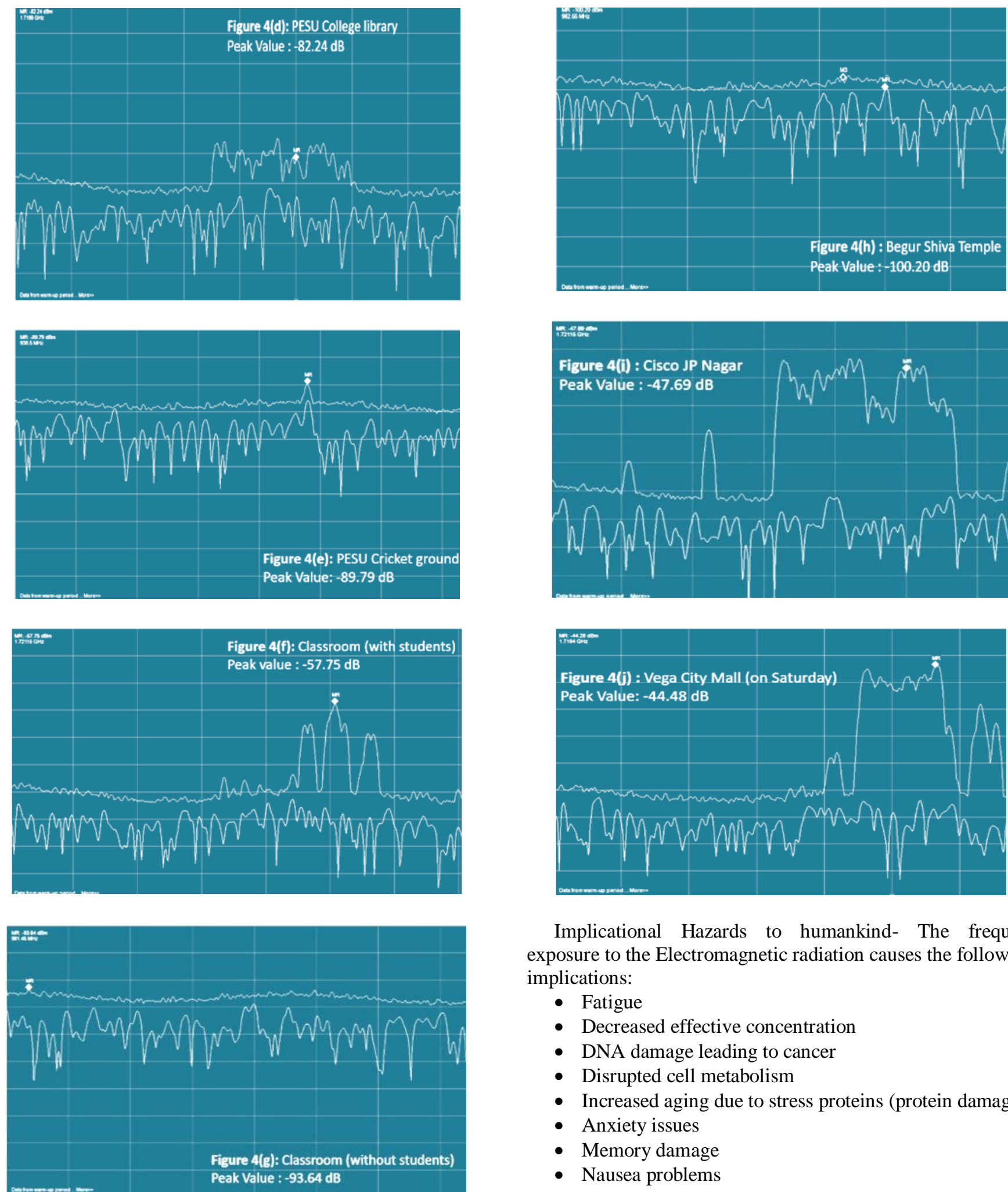

Implicational Hazards to humankind- The frequent exposure to the Electromagnetic radiation causes the following implications:

- Fatigue

- Decreased effective concentration

- DNA damage leading to cancer

- Disrupted cell metabolism

- Increased aging due to stress proteins (protein damage)

- Anxiety issues

- Memory damage

- Nausea problems

- Cornea damage 


\section{International Journal of Engineering Applied Sciences and Technology, 2020 \\ Vol. 5, Issue 4, ISSN No. 2455-2143, Pages 437-441 \\ Published Online August 2020 in IJEAST (http://www.ijeast.com)}

\section{PRECAUTIONS TO ALLEVIATE HARMFUL EFFECTS OF EMR}

Mobile users are urged to keep the mobile phones at a safe distance of $30-40 \mathrm{~cm}$ as the radiation intensity decreases with the distance. Thus, it is better to hold it farther away from the head so as to minimize the impacts of radiation

Calls' duration has to be taken care by the user of the mobile phone as the higher duration of mobile calls cause a higher radiation hazard.

Utilization of the safety materials-Canopies, curtains, enclosures can be manufactured from mesh type shielding fabrics. These are the type of fabrics that shield over a broad range of spectra. They can also be made with bamboo fibre and silver that are used for- bedding, drapes, clothing, grounding, tents.

Avoid the utilization of phones when the battery is low as the radiation is emitted at a higher rate during low battery. They should be used in areas that have a good reception of the signals, so that the transmit signals consumes less power. The utilisation of mobile phones in elevators, cars, planes etc must be minimised, as the radiation emitted is high within a closed environment.

Avoid exposure to high radiation areas such as a mobile tower etc. where the EM waves are highly persistent. Studies have shown correlation between an increased risk of cancer and high and frequent exposure to high levels of radiation exposure.

Essential EMI shielding articles must be incorporated into daily life when utilizing computers, mobile phones etc. A good example is the utilization of PANI based synthesis polymer EMI shielding fabrics (TRAI, 2014).

\section{CONCLUSION}

The radiation has affected the entire population already on a drastic level, and the advancement in technology is further increasing the level of EMR. Hence, we should work together in order to reduce the growth of these radiation activities. There is a drastic change in the situations which are related to severe and negative health effects due to exposure to electromagnetic fields that is becoming clearer and more difficult to ignore. It is hard to find cleaner and healthier alternatives, unless the public dedicate considerable amount of time into these issues and take initiative to learn and educate themselves as well as others about the EMF and EMR. The average level of EMF that we experience today is being estimated to be 50 times greater than what our previous generation had experienced at the same age that we are now. So, should the upcoming generations experience 50 times or even more electromagnetic fields than we do now? Steven Magee once said "Human mind and body metals appear to be lost through radio frequency exposure and I call this hypothesis: Internal Human Corrosion".

\section{ACKNOWLEDGEMENT}

The authors acknowledge Vision Group on Science and Technology, Government of Karnataka, Bangalore, for providing financial assistance in the form of a research study and the PES Institute of Technology, Bangalore South Campus, Bangalore for extending all support and encouragement in this study. The authors express their profound thanks and acknowledge all the authors and contributors of research articles and other reports which has been referred and cited in this research article. Also, the authors thank the editorial of Hillgrove Research Pvt. Ltd., Coimbatore, for their editorial service.

\section{REFERENCES}

1) Indira Nair, $M$ Granger Morgan, $H$ Keith Florog,"Biological Effects of Power Frequency Electric and Magnetic Fields - Background paper", IEEE Engineering in Medicine and Biology Magazine, September 1989, Vol. 8, Issue 3, DOI: 10.1109/51.35578

2) Manoj V, Naveen Kumar A, Revanasiddappa M, "EMF Pollution - Causes, Effects and Protection (Case study)", Recent Innovations in Science and Engineering - RISE 2016, Conference Proceedings. 30th April 2016

3) Telegram Regulatory Authority of India (TRAI) on Effects of Electromagnetic Field Radiation from Mobile Towers and Handsets" - Information paper No: 01/2014 QoS

4) https://environmentofearth.wordpress.com/2012/04/19/ele ctromagnetic-pollution/

5) https://in.tek.com/datasheet/rsa306-usb-real-timespectrum-analyzer-datasheet-0

6) https://signalhound.com/news/what-you-need-to-knowabout-real-time-spectrum-analysis/

7) https://ntp.niehs.nih.gov/ 